\title{
The distribution of attention within objects in multiple-object scenes: Prioritization by spatial probabilities and a center bias
}

\author{
CARY S. FERIA \\ San Jose State University, San Jose, California
}

\begin{abstract}
This study investigates how the attentional distribution within objects is affected by spatial probabilities, bias toward objects' centers (Alvarez \& Scholl, 2005), and object motion. In a multiple-object tracking task, observers tracked line objects while simultaneously detecting probes appearing on the objects. Experiments 1 and 2 manipulated the probabilities of probes appearing at the centers and ends of objects. Overall, probe detection was better at centers than at ends, but it was also affected by probe location probabilities; when probe locations were $100 \%$ certain, the center advantage was eliminated. Experiment 3 manipulated rotational, translational, and size-change components of object motion. The center advantage still occurred with stationary objects, and its magnitude was not affected by different motion types. These results indicate that attention is biased toward the centers of objects in multiple-object scenes, both for stationary and moving objects. They also imply that attentional prioritizations based on spatial probabilities can accompany moving objects.
\end{abstract}

In daily life, our visual system is often called upon to keep track of objects that are moving in the world around us. Automobile drivers tracking surrounding vehicles and athletes tracking opponents on the field, for instance, are readily able to maintain representations of their targets as they move in space and time. Studies have shown that human observers can track up to five objects with good accuracy, even if the objects being tracked are surrounded by identical untracked distractor objects (e.g., Pylyshyn \& Storm, 1988). Previous research has examined how attention is allocated among moving objects, such as when objects are separated in depth (Liu et al., 2005; Viswanathan \& Mingolla, 2002), are perceptually grouped (Suganuma \& Yokosawa, 2006; Yantis, 1992), or disappear briefly (Horowitz, Birnkrant, Fencsik, Tran, \& Wolfe, 2006; Keane \& Pylyshyn, 2006; Scholl \& Pylyshyn, 1999). However, less is known about the allocation of attention within each of the tracked objects. In this article, we investigate the spatial distribution of attention within tracked moving objects.

In a typical multiple-object tracking study, observers track a subset of small target items, such as circles, moving among identical distractors (e.g., Pylyshyn \& Storm, 1988). Only a few studies have examined tracking of extended objects, although these objects may be more naturalistic. vanMarle and Scholl (2003) found that, when the objects to be tracked were not solid, discrete items, but instead were nonrigid substances that "poured" from one location to another, tracking performance declined substantially. They concluded that the reduced ability to track these objects was due to the extension and contraction of the objects, which made it impossible to unambiguously pinpoint the location of each one. Scholl, Pylyshyn, and Feldman (2001) asked observers to track target points that were connected with distractor points. For instance, in one condition, each target point and distractor point were connected by a line to form an object, although both points moved independently. Tracking performance was impaired, leading Scholl et al. to conclude that attention spread throughout the entire line object, causing the observer to lose track of which end was the target.

Alvarez and Scholl (2005) were the first to examine the distribution of attention within each tracked object. In their study, observers tracked three target lines among three distractor lines. Intermittently during the motion, small probe dots appeared briefly at different locations along the target and distractor lines. Observers were asked to detect these probes, and the accuracy of detection of the probes at different locations was used as a measure of the distribution of attention across the lines. The results indicated that attention was concentrated at the centers of the lines during tracking. In addition, the longer the lines were, the greater the center advantage.

Why does attention concentrate at the centers of moving objects being tracked? Alvarez and Scholl (2005) suggested that the visual system has an inherent attentional bias toward the centers of moving objects, and this hypothesis seems viable, in view of research showing biases in vision toward the centers of objects, arrays, and scenes. Several studies have shown that when a sacca- 
dic eye movement is directed to an extended object, the line of sight lands, on average, near the object's center of gravity (see, e.g., Vishwanath \& Kowler, 2003, 2004). Similarly, when two or more targets are presented in close proximity, the initial saccade is deviated toward the center of gravity of the configuration (e.g., Coëffé \& O'Regan, 1987; Findlay, 1982). It is generally believed that saccade landing positions are computed by averaging across the structure of the target object or the array of target objects (e.g., Vishwanath \& Kowler, 2004). The center of gravity of an object is also used in perceptual localization tasks; when observers perceptually align spatially extended targets, the reference point upon which the alignment is based tends to coincide with the centroid of the luminance or contrast distribution of the target (e.g., Morgan, Hole, \& Glennerster, 1990; Whitaker, McGraw, Pacey, \& Barrett, 1996; Whitaker \& Walker, 1988). Other researchers have noted the role of the center of gravity in visual attention. Grabowecky, Robertson, and Treisman (1993) used a visual search paradigm with unilateral neglect patients and found evidence that the reference frame of attention orients to the center of gravity of the elements in the display. Zhou, Chu, Li, and Zhan (2006) demonstrated in a spatial-cuing experiment that the center of a scene attracts attention. In Zhou et al.'s study, observers were presented with four small boxes arranged in a square formation and a fifth box at the center of this array. The observers fixated to one side of the display; a cue then appeared at one of the five boxes, and a target appeared at another of the boxes. The cue location was not predictive of the target location. Zhou et al.'s results showed target detection to be faster at the center box than at all other locations, demonstrating an attentional bias toward the center of the scene.

An alternative explanation for Alvarez and Scholl's (2005) finding of attentional concentration at the centers of moving objects is that the concentration on the centers is due to the context provided by the probes. Several studies have shown that the visual system attentionally prioritizes locations on the basis of the probabilities of a target appearing at the locations. Targets in high-probability locations are detected more efficiently than targets in low-probability locations, which suggests that the visual system strategically allocates its resources to the locations most likely to contain a target (see, e.g., Geng \& Behrmann, 2005; Prinzmetal, McCool, \& Park, 2005; Walthew \& Gilchrist, 2006). Although most of these spatial prioritization studies have dealt with visual search for letters in simple arrays, a few have examined spatial prioritizations within objects and object-like configurations. Miller (1988) presented observers with a configuration of horizontally aligned letters in which a target letter could occur with high or low probability at each of four locations. After practice, the configuration was shifted in its spatial location to the left or right, so that the high- and low-probability locations were at new screen locations. Target detection was facilitated at the high-probability location relative to the configuration, demonstrating that spatial prioritizations can occur within a configurationbased reference frame. Hoffmann and Kunde (1999) extended this finding to differently shaped configurations of letters. In a study examining the relationship between spatial prioritization and object-based attention, Shomstein and Yantis (2004) presented observers with a pair of rectangle objects. After a cue appeared at one end of one of the rectangles, a target letter appeared with high or low probability at some location either within that rectangle or within the other. The results showed that location probabilities, as well as a same-object advantage, affected detection of the target letter. Coëffé and O'Regan (1987) and He and Kowler (1989) showed that saccade landing positions are also affected by target location probabilities.

Thus, Alvarez and Scholl's (2005) finding that attention is concentrated at the centers of objects could be due to prioritization by spatial probabilities. In Alvarez and Scholl's first experiment, probes were more likely to occur at the center of each object than at either of its ends, so observers might have strategically attended to the center in order to better detect the probes. Alvarez and Scholl began to address this issue in their second experiment, in which the probability of probes occurring at the centers of objects was reduced to 2 , whereas the probability of their appearance at either end was increased to .4. Nonetheless, the results showed that attention was still concentrated on the objects' centers to the same extent as in the first experiment. However, a limitation of this manipulation was that the distribution of probes was still symmetrical about the center of the line, and thus, attention focused on the center would still be advantageous for detecting probes on both sides of the line. Observers might not be readily able to split attention between two separate points on an object while allocating less attention to areas between those two points. On the other hand, if a single location on an object is the one with the highest probability, this location might be afforded an attentional advantage, whether or not it is the center of the object.

If spatial probabilities modulate the center bias, another question of interest is whether the center bias still occurs when probe locations are known with certainty. Previous research has shown that when observers are in a highly focused attentional state, attentional allocation is not affected by some factors that normally do affect the attentional distribution. For instance, Yantis and Jonides (1990) found that although abrupt stimulus onsets usually capture attention, they do not capture attention when it is being directed to a location indicated by a completely valid cue. In a study by Shomstein and Yantis (2002), observers identified a target letter while attempting to ignore flanking distractors that appeared either within the same object as the target or within a different object. The results showed that when target location could vary, flankers were more disruptive when they occurred on the same object as the target than when they occurred on the other object. Yet, when the target location was constant, flankers on the same object and on the other object were equally disruptive. This suggests that when target location is uncertain, the entire relevant object is given enhanced attentional priority, but when target location is certain, other locations on the object are not given enhanced attentional priority. However, it should be noted that Chen and Cave (2006) suggested that, in Shomstein and Yantis's (2002) study, the 
observers did not interpret the stimulus pattern as being two separate objects. Chen and Cave's study showed that when active top-down interpretation of the configuration encourages observers to interpret the stimulus as two separate objects, object-based flanker effects do occur, even when the target location is known with certainty.

If the visual system has an inherent bias toward the centers of moving objects, what aspects of the stimulus are necessary for the bias to occur? Objects can undergo several types of motion, including rotation, translation, and size changes. What types of motion will produce the center bias? Is attention concentrated at the centers of objects even when the objects are stationary? A first hypothesis comes from Alvarez and Scholl (2005), who suggested that attention might track the most stable point on an object. In their experiments, because the endpoints of each line moved randomly and independently, the center of the line was consistently the most stable point on the object, with the slowest average speed and the fewest changes in direction of any point on the line. According to this hypothesis, when objects move in such a way that the center is not the most stable point, the center will not be attended more than other points on the object. Rotational motion and size changes contribute to the center being the most stable point on the object, whereas translational motion does not. Thus, if Alvarez and Scholl's stability hypothesis is correct, when the objects being tracked do not rotate or change size, attention will not be concentrated at their centers. A second hypothesis is that attention is drawn to the center of any object that is moving from one location to another. In accordance with this hypothesis, even if both rotational motion and size changes are removed, and objects move in simple translation, the center bias will still occur. This would suggest that the center bias applies to most situations that would be considered instances of multiple-object tracking. A final hypothesis is that attention to the centers of objects is unrelated to motion, and that attention is concentrated at the centers of objects even if they are not moving. This would be consistent with the findings, discussed above, that a bias toward centers is prevalent in many perceptual and attention tasks (e.g., Vishwanath \& Kowler, 2003, 2004; Zhou et al., 2006).

In the present study, I assessed the distribution of attention within moving objects and examined how this distribution is affected by task demands, produced by either probe probabilities or different types of object motion. Experiment 1 focused on whether the distribution of attention within moving objects is prioritized by spatial probabilities. Observers tracked moving line targets among identical distractors while detecting brief probes on the target lines. In one condition, probes were much more likely to occur at the center of an object than at either end. In the other condition, probes were much more likely to occur at one end than at either the center or the opposite end. The results of this experiment showed that the attentional distribution is affected by prioritization by spatial probabilities and by a center bias. Experiment 2 set out to examine whether the center concentration occurs even when probe locations are known with $100 \%$ certainty. Probes occurred either at an object's center with probability 1 , or at one of its endpoints with probability 1 . The results showed that when there is no uncertainty regarding the probe location, the center bias is eliminated. Experiment 3 investigated the contributions of rotational motion, translational motion, and size changes in producing the center bias. A factorial design examined all combinations of the presence and absence of these three types of motion. The results showed that the center bias occurs regardless of the types of motion present and that attention is biased even toward the centers of stationary objects.

\section{EXPERIMENT 1}

The purpose of Experiment 1 was to determine how the distribution of attention over multiple moving objects is affected by spatial probabilities. Specifically, it was designed to ascertain whether the visual system is inherently biased to attend to the centers of objects, or whether this system attentionally prioritizes locations on objects on the basis of task demands, such as the spatial probabilities of probes. Alvarez and Scholl (2005) found that observers attended more to the centers of objects, but in their design this could have been due to the task demand to attend to the high-probability location, which was also the center of the object. Thus, the purpose of Experiment 1 was to tease apart these two factors.

The observers here tracked two target lines among two identical distractors (see Figure 1). The lines changed in length and orientation as they moved. Intermittently during the motion, gray circular probes appeared briefly at either the center of a line or near one of its endpoints. Observers detected these probes by pressing a key on the keyboard. At the end of the motion, they identified which of the lines were the original targets by selecting them with the mouse.

Each line was drawn in two colors: two thirds of the line was one color, and the other third was another color. In one block of trials, the probes were more likely to appear at the center, with a probability of occurrence of .8 at the center, .1 at the different-color end, and .1 at the same-color end. In the other block of trials, the probes were more likely to appear at the different-color end, with a .8 probability of occurring at that end, as opposed to a .1 probability of appearing at either the center or the same-color end. The use of two colors was necessary so that observers could distinguish which end had the high probability of probe appearances in this condition.

If the visual system has an inherent attentional bias to the centers of objects, observers should have better probe detection performance for probes occurring at the centers of objects, rather than at either of the ends. This pattern should occur regardless of the probabilities of probes occurring at these locations. Alternatively, the visual system may strategically allocate its resources to the locations on an object that are most likely to contain a probe (see, e.g., Geng \& Behrmann, 2005; Prinzmetal et al., 2005). According to this account, a probe probability manipulation should affect the pattern of probe detection rates. Specifically, when the high-probability location is the center, observers should have better detection performance there 
A

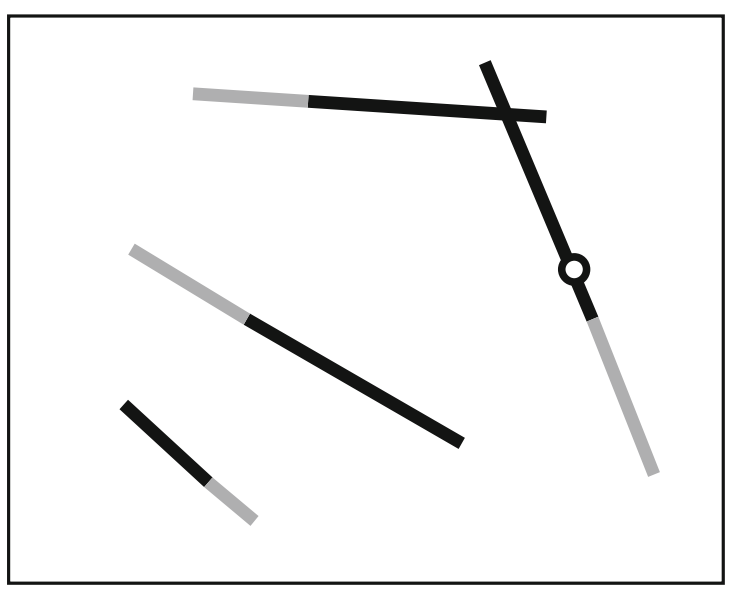

B

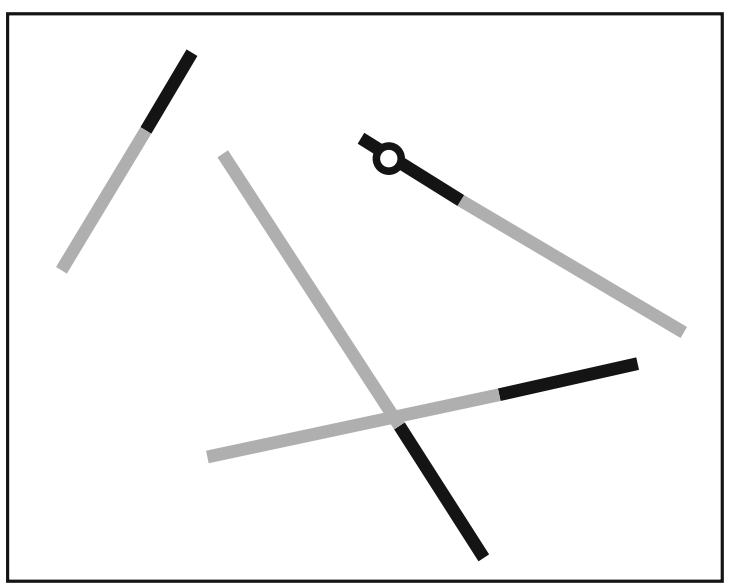

Figure 1. Examples of the stimuli used in Experiments 1 and 2 (not drawn to scale). (A) Lines with a center probe. (B) Lines with a different-color end probe.

than at either of the ends, but when the high-probability location is the different-color end, they should have better detection performance at that end than at either the center or the same-color end.

\section{Method}

Observers. The observers for this experiment were 20 undergraduate students from Morehead State University in Kentucky. Each participated in one, hour-long, session and was compensated with course credit. All observers had self-reported normal or correctedto-normal vision, and none of them was familiar with the purposes of the experiment.

Apparatus. The observers were seated in a darkened room approximately $44 \mathrm{~cm}$ from the display. The stimuli were presented on a 20 -in. (50-cm) flat-screen CRT monitor with a pixel resolution of 2,048 $\times$ 1,536 , controlled by a Dell Dimension workstation. The experimental procedure was generated in $\mathrm{C}++$ using the OpenGL libraries.

Stimuli. On each trial, four lines were presented on a black background (see Figure 1). Each line had a width of $0.02^{\circ}$ of visual angle
(1 pixel). At the beginning of each trial, eight points in the display area were chosen randomly, and pairs of the points were connected to form four lines. Two of the four lines were chosen as targets. These two lines flashed off and on five times over a period of $2.5 \mathrm{sec}$. Then the motion phase began, lasting for $20 \mathrm{sec}$. During this time, each endpoint of each line moved independently, and thus both the length and the orientation of the lines varied. At the beginning of each trial, each endpoint was independently assigned a speed between $0.43^{\circ}$ and $1.19^{\circ}$ per second in both the horizontal and vertical directions, so that each endpoint's overall velocity was between $0.61^{\circ}$ and $1.68^{\circ}$ per second. The speed of each endpoint remained constant throughout the trial, and the lines could intersect each other. The edges of the display area were bounded by a gray outline rectangle that subtended $19^{\circ} \times 15^{\circ}$. The lines composing the rectangle were $0.05^{\circ}$ in width ( 2 pixels). There was a buffer of width $0.2^{\circ}$ (10 pixels) around each line of the rectangle, so that the line segments would not touch the rectangle. Whenever an endpoint reached the edge of the display, its direction was reversed to send it back into the display area.

During the motion phase, several gray probe disks were presented on the lines. Each probe had a diameter of $0.14^{\circ}$ and was presented for $215 \mathrm{msec}$. Only one probe was presented at a time, and a random interval of 1,000 to $2,200 \mathrm{msec}$ passed between the onsets of one probe and the next. The first probe appeared between 1,000 to $2,200 \mathrm{msec}$ after the motion began, and the last appeared at least $1,000 \mathrm{msec}$ before the end of the motion. Thus, between 8 and 19 probes could be presented on each trial.

The probes appeared only on target lines. The locations in which probes could appear were at the center of a line and near either of the endpoints. Endpoint probes were inset $0.14^{\circ}$ (one probe diameter) toward the center of the line.

Each line was drawn in two colors, light green and light blue. For 10 of the observers, two thirds of each line was blue, and the other third was green. This pattern held for every line these observers saw on every trial. For these observers, same-color end probes were probes that occurred at the blue end (which was the same color as the center portion of the line), and different-color end probes were those that occurred at the green end. For the other 10 observers, two thirds of each line was green, and the other third was blue. For these observers, same-color end probes were those occurring at the green end, and different-color end probes were those occurring at the blue end. The use of two colors was necessary so that observers could distinguish which end of a line had the high probability of probe appearances in each condition.

The probe probability conditions were presented within subjects, in separate blocks. In one block (center high-probability location), the probes appeared at the center with probability .8 , at the different-color end with probability .1 , and at the same-color end with probability .1 . In the other block (different-color-end highprobability location), the probes appeared at the center with probability .1, at the different-color end with probability .8 , and at the same-color end with probability .1. The order of these conditions was counterbalanced.

Procedure. Observers were instructed to track the target lines during the motion phase and to watch for the appearances of probes on target lines. The observers were instructed to treat the tracking task as primary and the probe detection task as secondary. Upon seeing a probe, they were to press a key as quickly as possible. Only keypresses within $1,000 \mathrm{msec}$ of a probe onset were counted as hits. All other keypresses were considered false alarms. The observers were informed of the probabilities of probes occurring in each of the locations on the lines.

At the end of the motion phase, the lines stopped moving, and the observer used the mouse cursor to select the two original target lines. The selected lines changed color, and the observers could undo and redo their selections if desired. After selecting their choice of lines, the observers pressed a key and were given feedback about their tracking accuracy (the number of target lines correctly selected) and probe detection accuracy (the number of probes detected out of the number of probes displayed). If five or more false alarms were made 
on a given trial, the observer was penalized by having to wait $30 \mathrm{sec}$ at this point, and the trial was considered invalid. After viewing the feedback, the observers pressed a key to move on to the next trial.

Design. The independent variables were probe location (center, different-color end, or same-color end) and high-probability location (center or different-color end). Both of these variables were run within subjects. In addition, the probe events were sorted into four line length categories: less than $4.3^{\circ}$ of visual angle (180 pixels), between $4.3^{\circ}$ and $8.4^{\circ}$ (181 and 350 pixels), between $8.4^{\circ}$ and $11.5^{\circ}$ (351 and 480 pixels), and greater than $11.5^{\circ}$ (480 pixels). These categories constituted another within-subjects independent variable in the analysis, line length (very short, short, long, or very long).

The dependent variable was the probe detection miss rate. This was defined as the number of probes that were not detected, divided by the number of probes presented. In the data analysis, each probe event was considered independently and without regard to the trial from which it came. Each observer completed two blocks, each of which consisted of 5 practice and 25 experimental trials. Within each block, the trials were presented in a random order for each observer.

\section{Results}

An average of 11.5 probes were presented per experimental trial, resulting in an average of 573 probes per observer. Line-tracking accuracy averaged $97.4 \%$ across all observers. Only trials on which (1) both of the target lines were correctly identified and (2) no more than four false alarms were recorded were included in the probe detection analyses. Thus, an average of 3.75 trials, or 42.4 probe events, were excluded per observer. An average of 0.93 false alarms occurred per tracking trial, for trials included in the probe detection analyses. The mean detection miss rate was calculated for each observer for each of the 24 conditions and was submitted to a 3 (probe location) $\times$ 2 (high-probability location) $\times 4$ (line length) repeated measures ANOVA.

The main effect of probe location was significant $[F(1.8,34.7)=44.85, p<.001]$. (Note: All tests of within-subjects independent variables with more than two levels were adjusted using the Greenhouse-Geisser pro- cedure, to compensate for possible deviations from sphericity.) Bonferroni post hoc tests were used to test for differences between the locations. The miss rates were lower for probes occurring at centers $(M=.39, S D=.15)$ than for those at different-color ends $(M=.60, S D=.11 ; p<$ $.001)$. Miss rates were also lower when probes occurred at centers rather than at same-color ends $(M=.65, S D=$ $.13 ; p<.001)$. Miss rates did not differ for the probes at different-color and same-color ends $(p>.05)$.

The main effect of high-probability location was not significant $[F(1,19)=0.11, p>.05]$, since miss rates did not differ in the different-color end $(M=.55, S D=.14)$ and center $(M=.55, S D=.09)$ high-probability conditions.

Importantly, a significant interaction was found between probe location and high-probability location $[F(1.9$, $36.2)=56.58, p<.001]$. This interaction can be seen in Figure 2. Bonferroni comparisons indicated that in the center high-probability condition, center probes had lower miss rates than did either different-color $(p<.001)$ or same-color $(p<.001)$ end probes, whereas differentcolor end probes did not differ from same-color end probes $(p>.05)$. However, in the different-color-end high-probability condition, center probes and differentcolor end probes did not differ in miss rates $(p>.05)$, although both had lower miss rates than did same-color end probes $(p s<.01)$.

The main effect of line length was significant $[F(2.5$, $47.5)=21.42, p<.001]$, in that miss rates increased as line length increased. A significant interaction was also found between probe location and line length $[F(3.9$, $74.0)=2.68, p<.05$ ], as well as a significant three-way interaction between probe location, high-probability location, and line length $[F(3.5,67.3)=3.18, p<.05]$. These interactions can be seen in Figure 2. In the center high-probability condition, miss rates for both differentcolor and same-color end probes increased with line length, whereas those for center probes did not. Yet, in the
High Probability: Center

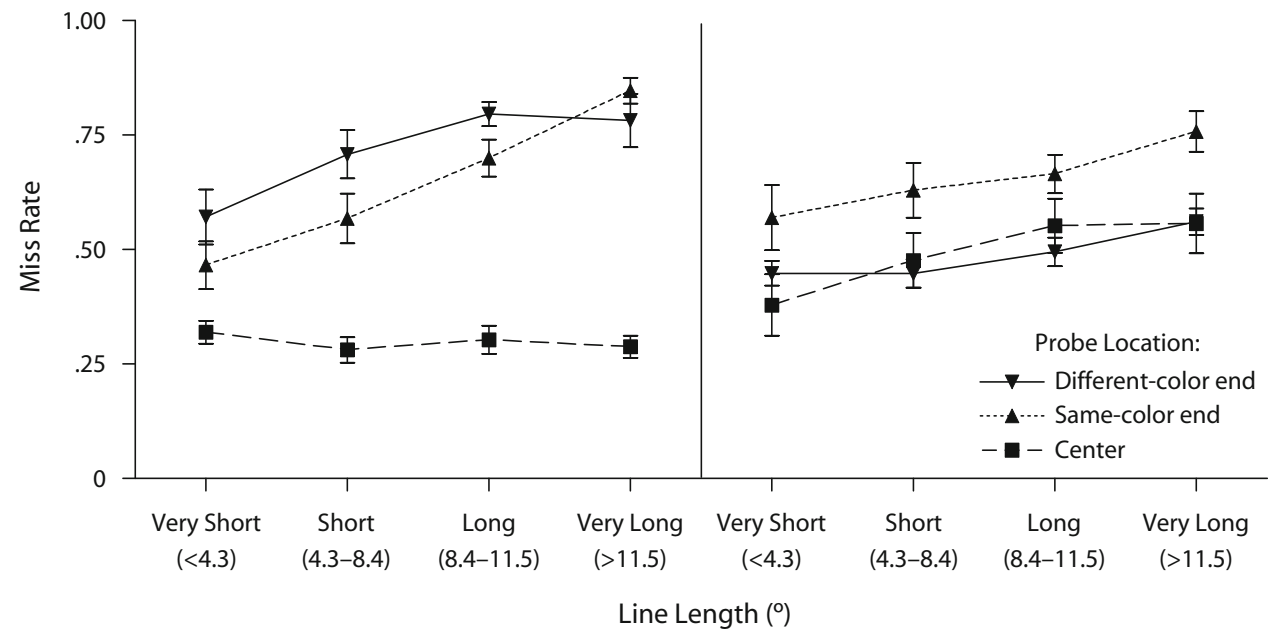

Figure 2. Miss rate as a function of line length, high-probability location, and probe location in Experiment 1. 
different-color-end high-probability condition, miss rates increased with line length for probes at all locations.

\section{Discussion}

Experiment 1 revealed two main results. First, probe detection performance was better, overall, at centers than at ends of lines. This result indicates that attention is biased toward the centers of moving objects being tracked and is consistent with that of Alvarez and Scholl (2005). Second, probe detection was enhanced at higher-probability locations. In other words, the effect of probe location was tempered by the probe probability distribution. When the center was the high-probability location, attention was focused there the most, but when the different-color end was the high-probability location, attention was focused equally on that end and the center. This finding indicates that the attentional distribution within moving objects being tracked is prioritized by spatial probabilities. Together, these results suggest that two factors influence the distribution of attention to moving objects: (1) an inherent center bias and (2) prioritization by spatial probabilities. The finding that, even when probes were most likely to occur at the differentcolor end, probe detection was not significantly better at the different-color end than at the center indicates that the probability manipulation was insufficient to eliminate the center bias. This suggests that the center bias found by Alvarez and Scholl was not simply due to the higher probability of the center location in their design.

However, even with the probe probability distributions used in Experiment 1, there may have been a strategic advantage for attending to the center. Focusing attention on the center means that both of the ends of the line are relatively close to the focus of attention, and thus probes occurring at either end can be detected fairly well. On the other hand, when attention is focused on one end of the line, the opposite end is quite far from the center of attention. Observers may choose to avoid focusing attention so far on one side of the line that probes on the opposite side will rarely be detected. This issue was addressed by the use of different probe probability distributions in Experiments 2 and 3.

Another finding of Experiment 1 was that, when the center was the high-probability location, detection performance declined at the endpoints but remained constant at the center as line length increased. This pattern differs slightly from the interaction found by Alvarez and Scholl (2005), in which detection performance declined at the endpoints and improved at the center as line length increased. Alvarez and Scholl interpreted this result as meaning that, as object size increases, attention becomes increasingly concentrated at the object's center. The present results are consistent with the idea that, when spatial probabilities prioritize attention to the center of the object, attention becomes more concentrated at the center of the object, relative to the endpoints, as object size increases. A new finding from the present Experiment 1 is that when the different-color end was the high-probability location, detection performance declined for probes at all locations as line length increased. This suggests that, when spatial probabilities prioritize attention to a location other than the center of the object, attention does not increasingly concentrate on any part of the object as object size increases.

It might be contended that observers may not have needed to track the targets throughout the entire trial, because the probes appeared only on targets, and thus served as reminders of which lines these were. Thus, observers could have focused solely on the probe detection task for the majority of the trial, and only begun to track the targets near the end. In order to address this possibility, a control experiment was run in which probes appeared on distractor as well as on target lines. The method of this experiment was otherwise identical to that of Experiment 1 , and 20 new observers participated. This control experiment necessitated tracking the targets constantly from the beginning of each trial, since there was no difference between targets and distractors once the motion began. Line-tracking accuracy averaged $94.2 \%$ across all observers, which was similar to that of Experiment 1. Lower miss rates were found on targets $(M=.62, S D=$ $.08)$ than on distractors $(M=.68, S D=.08)[F(1,19)=$ $10.10, p<.01]$. Separate ANOVAs were also performed for targets and for distractors. For targets, the main effect of probe location was significant $[F(1.9,35.6)=15.49$, $p<.001]$, as was the probe location $\times$ high-probability location interaction $[F(1.7,32.1)=10.56, p<.01]$. The patterns of results for the target objects were similar to those found in Experiment 1, confirming that attentional center bias and prioritization by spatial probabilities did occur during target tracking. For distractors, the main effect of probe location $[F(1.9,35.4)=5.38, p<.05]$ and the probe location $\times$ high-probability location interaction $[F(1.8,34.2)=5.00, p<.05]$ were also both significant. These results demonstrate that center bias (see also Alvarez \& Scholl, 2005) and prioritization by spatial probabilities affected the attentional distribution within untracked distractor objects as well as within targets.

\section{EXPERIMENT 2}

Experiment 1 demonstrated a strong bias to attend to the centers of moving objects. That bias was tempered, although not eliminated, by task demands in the form of spatial probabilities. Yet, does the center bias persist when the task requires attention to be allocated only to one particular location on the object? In other words, if the task requires attention solely to the end of the line, will the motion of the line still draw attention toward the center? It may be that, to track a moving object, one must attend to its center. If this is the case, then even if probes occur only on one end of the line, attention would still have to focus to some extent on the center of the line. This hypothesis receives some support from Alvarez and Scholl's (2005) Experiment 3, in which observers were instructed to track only one endpoint of each line, rather than the entire line. Although the only locations relevant for the tracking were these target endpoints, this experiment found that attention was still concentrated at the lines' centers. However, in that experiment, probes appeared at both endpoints of each line and at the center, so task demands from the spa- 
tial probabilities may still have encouraged attention to the center.

On the other hand, research has shown that some factors that usually affect attentional distributions, such as abrupt stimulus onsets (Yantis \& Jonides, 1990) and object-based attention (Shomstein \& Yantis, 2002), have no effect when observers are in a highly focused attentional state. These findings suggest that when probe locations are known with $100 \%$ certainty, attention might not be drawn to the center of the line.

In Experiment 2, during one block of trials, probes always appeared at the center, and during another block, the probes always appeared at the different-color end. If an observer must focus attention on the center of a moving object in order to track it, then detection performance should be better for probes at the center than for those at the end. On the other hand, if observers are not biased toward objects' centers when they are in a highly focused attentional state, because they know the probe locations with certainty, detection should then be equal for probes at the ends and centers.

\section{Method}

Observers. The observers were 16 undergraduate students from Morehead State University. Each participated in one, hour-long, session and was compensated with course credit. All observers had self-reported normal or corrected-to-normal vision, and none was familiar with the purposes of the experiment.

Apparatus. The apparatus was the same as in Experiment 1, except that the observers were seated approximately $114 \mathrm{~cm}$ from the display.

Stimuli. The stimuli were the same as in Experiment 1, with the following exceptions. Each line had a width of $0.01^{\circ}$ of visual angle (1 pixel). At the beginning of each trial, each endpoint was independently assigned a speed between $0.17^{\circ}$ and $0.49^{\circ}$ per second in both the horizontal and vertical directions, so that each endpoint's overall velocity was between $0.24^{\circ}$ and $0.69^{\circ}$ per second. The display area subtended $15^{\circ} \times 12^{\circ}$. The lines composing the gray outline rectangle defining the boundaries of the display area were $0.02^{\circ}$ in width ( 2 pixels), and the buffer around each line of the rectangle had a width of $0.1^{\circ}$ (10 pixels). Each probe had a diameter of $0.07^{\circ}$. A random interval of 2,000-4,000 msec passed between the onsets of one probe and the next. The first probe appeared between 2,000 and $4,000 \mathrm{msec}$ after the motion began, so that between four and nine probes could be presented on each trial. Endpoint probes were inset $0.07^{\circ}$ (one probe diameter) toward the center of the line.

The color schemes of the lines were the same as in Experiment 1. However, each observer viewed one color scheme during the first block and the other during the second block. Thus, the color of the section of a line on which the probes appeared remained constant across the two blocks. Eight of the observers saw probes appear on the blue section of the line in both blocks, and the other 8 saw probes appear on the green section of the line in both blocks.

The probe location conditions were presented within subjects, in separate blocks. In one block (center probe location), the probes always appeared at the center. In the other block (different-color end probe location), the probes always appeared at the different-color end. The order of these conditions was counterbalanced.

Procedure. The procedure was the same as in Experiment 1.

Design. The design was the same as in Experiment 1, with the following exceptions. The independent variable was probe location (center or different-color end), which was run within subjects. In addition, the probe events were sorted into four categories of line length: less than $3.5^{\circ}$ of visual angle ( 360 pixels), between $3.5^{\circ}$ and $6.8^{\circ}$ (361 and 700 pixels), between $6.8^{\circ}$ and $9.3^{\circ}$ (701 and 960 pix- els), and greater than $9.3^{\circ}$ (960 pixels). These categories comprised another within-subjects independent variable, line length (very short, short, long, or very long), in the analysis.

\section{Results}

An average of 5.9 probes were presented per experimental trial, resulting in an average of 297 probes per observer. Line-tracking accuracy averaged $97.3 \%$ across all observers. Only trials on which (1) both of the target lines were correctly identified and (2) no more than four false alarms were recorded were included in the probe detection analyses. Thus, an average of 2.94 trials, or 17.6 probe events, were excluded per observer. An average of 0.80 false alarms occurred per tracking trial, for trials included in the probe detection analyses. The mean detection miss rate was calculated for each observer for each of the eight conditions and was submitted to a 2 (probe location) $\times 4$ (line length) repeated measures ANOVA. Figure 3 shows the mean miss rates for each condition in Experiment 2.

The main effect of probe location was not significant $[F(1,15)=0.82, p>.05]$ : Miss rates did not differ for probes occurring at centers $(M=.47, S D=.18)$ and at ends $(M=.45, S D=.19)$. The main effect of line length was significant $[F(2.4,35.5)=7.75, p<.01]$, with miss rates increasing as line length increased. Probe location and line length did not significantly interact $[F(2.0$, $30.0)=0.82, p>.05]$.

\section{Discussion}

In Experiment 2, probe detection performance was equal for probes occurring at the centers and ends of lines. This result demonstrates that when probe locations are known with $100 \%$ certainty, the center bias is eliminated. This indicates that it is not mandatory for attention to be concentrated at the center of a moving object in order to track the object.

An additional finding of Experiment 2 was that, for probes occurring at both centers and ends, detection performance declined as line length increased. This result is

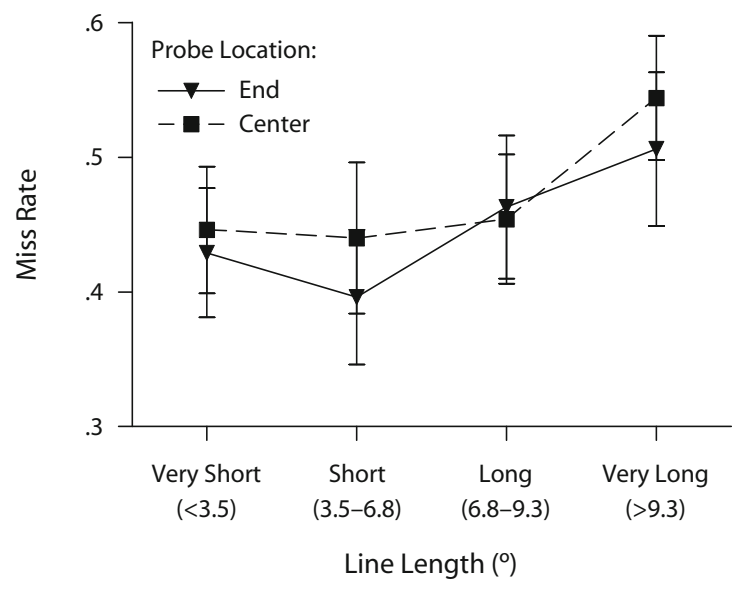

Figure 3. Miss rate as a function of line length and probe location in Experiment 2. 
somewhat surprising: Because observers knew with $100 \%$ certainty the locations on objects at which the probes would appear, they might have been expected to attend only to those locations, and thus not to be affected by increasing line length. That expectation would be consistent with Shomstein and Yantis's (2002) finding that, when the target location on an object is known with $100 \%$ certainty, other locations on the object are not given enhanced attentional priority. One possible explanation for this finding of worse detection on longer lines relates to the distribution of attention; perhaps it is distributed over multiple locations on the line, with the amount of attention available for any one point decreasing as the length increases. Observers might be unable to restrict their attention solely to the relevant probed location while tracking multiple moving objects. Perhaps Shomstein and Yantis's (2002) finding that attention is restricted to the target location is only true in the case of stationary objects, whereas the tracking of objects forces attention to spread to other locations on the object. This hypothesis is consistent with Scholl et al.'s (2001) finding that tracking performance was worse when tracking a target point that was connected by a line to a distractor point than when tracking an isolated target point. In the present study, the spread of attention to irrelevant locations on a moving object might be due to the increased difficulty of pinpointing the exact probed location when an object is moving. However, an alternative explanation is that detection was worse for longer lines not because of any characteristic of the attentional distribution, but rather because a probe produces a smaller luminance change relative to the line as a whole on a longer line than on a shorter one.

\section{EXPERIMENT 3}

Although Experiment 2 indicated that the attentional bias toward the centers of moving objects can be eliminated under $100 \%$ certainty of the probe location, Experiment 1 demonstrated that when uncertainty is present, the center bias does occur, and that the center bias is not due solely to probe location probabilities. If spatial probabilities are not the cause of the center bias, then what does produce it? Previous research assumed that the root of the center bias is the motion of the objects (Alvarez \& Scholl, 2005). The purpose of Experiment 3 was to investigate whether the center bias is indeed caused by the tracking of motion, and if so, to determine what types of motion will produce it. Experiment 3 examined the effects of rotational, translational, and size-change motion components on the center bias. A factorial design examined all combinations of the presence and absence of these three types of motion.

Alvarez and Scholl (2005) hypothesized that the observer attends to the most stable point of an object. According to this hypothesis, an attentional center bias would be expected when objects rotate about their centers or change in size, because in these cases the center is more stable than other points on the line. No attentional center bias should occur, however, when objects undergo straight-line translational motion or are stationary, because in these cases the center is no more stable than other points.

A second hypothesis is that attention is drawn to the center of any moving object, regardless of the type of motion it is undergoing. A final hypothesis is that attention to the centers of objects is unrelated to motion, so that attention is concentrated at the centers of objects even if they are not moving. Studies on saccadic eye movements (e.g., Vishwanath \& Kowler, 2003, 2004) have found that saccades to stationary objects or arrays land near the centers of the objects or arrays, and Zhou et al. (2006) found an attentional bias toward the center of a scene. Together, this research suggests that a center bias may be a pervasive phenomenon in visual perception. According to this hypothesis, the attentional center bias should occur regardless of which motion types are present, and should be present even when objects are completely stationary.

To avoid any strategic advantage for attending to the centers of objects, Experiment 3 used probe probabilities of .5 at the center and .5 at the designated end of objects. As noted in the Experiment 1 Discussion, when probes appear at both ends of a line, observers have an incentive to attend to the center, because if one end is the attentional focus, it will be difficult to detect probes occurring as far away as the opposite end of the line. In the probe probability distribution used in Experiment 3, because the probes never appeared at the opposite end of the line, there was no strategic incentive to attend to the center.

\section{Method}

Observers. The observers were 48 undergraduate students from Morehead State University. Each participated in one, hour-long, session and was compensated with course credit. All observers had selfreported normal or corrected-to-normal vision, and none of them was familiar with the purposes of the experiment.

Apparatus. The apparatus was the same as in Experiment 1, except that the observers were seated approximately $114 \mathrm{~cm}$ from the display.

Stimuli. The stimuli were the same as in Experiment 1, with the following exceptions. Each line had a width of $0.01^{\circ}$ visual angle ( 1 pixel), on a display area subtending $15^{\circ} \times 12^{\circ}$. The lines composing the gray outline rectangle defining the boundaries of the display area were $0.02^{\circ}$ in width ( 2 pixels). The buffer around each line of the rectangle had a width of $0.1^{\circ}$ (10 pixels). Each probe had a diameter of $0.08^{\circ}$, and the endpoint probes were inset $0.07^{\circ}$ (one probe diameter) toward the center of the line.

The factorial design, which is described in more detail in the Design subsection below, included the independent variables rotation (present [RP] or absent [RA]), translation (present [TP] or absent [TA]), and growth (present [GP] or absent [GA]). Thus, there were eight motion conditions: RP-TP-GP, RP-TP-GA, RPTA-GP, RP-TA-GA, RA-TP-GP, RA-TP-GA, RA-TA-GP, and RA-TA-GA. At the beginning of each trial, in the growth-present conditions, each line was randomly assigned a growth speed of either $0.97 \% \mathrm{sec}$ (growth) or $-0.97 \% \mathrm{sec}$ (shrinking). Additionally, in the translation-present conditions, each line was assigned a translation speed of $1.30 \% \mathrm{sec}$. The $x$ - and $y$-components of the translation speed were chosen randomly, with the constraint that both the $x$ - and $y$-components had to be at least $0.26^{\circ} / \mathrm{sec}$. Finally, in the rotationpresent conditions, each line was assigned a rotation speed of $0.16^{\circ} \%$ sec (degrees here refers to angle of rotation). Each of these speed components remained constant throughout the trial. Whenever an endpoint reached the edge of the display, its growth direction was reversed (in the growth-present conditions), its translation direc- 
tion was reversed (in the translation-present conditions), and its rotation direction was reversed (in the rotation-present conditions). The length of the lines was always between $1.94^{\circ}$ and $11.66^{\circ}$. In the growth-present conditions, whenever a line grew to a length of $11.66^{\circ}$, it switched to shrinking, and whenever a line shrank to a length of $1.94^{\circ}$, it switched to growing.

Each line was drawn in three colors: One third of the line was light blue, one third was green, and one third was purple. There were six color schemes, consisting of all possible combinations of the three colors in the three sections of the line. Eight of the observers saw each color scheme, and the color scheme remained constant for every line on every trial. Three colors were used in Experiment 3, rather than two colors as in Experiments 1 and 2, because in Experiments 1 and 2 the high-probability end was also always the different-color end. In those experiments, it was possible that the distinct coloring of the high-probability end could have produced greater ease (or greater difficulty) in detecting the probes. In Experiment 3, having three differently colored areas of equal size avoided any potential bias of directing attention to a larger or smaller colored area.

Observers were informed that probes would only occur at the center and at one of the ends of the lines, and they were informed which color the designated end would be. The probes appeared at the center or at the designated end with probability .5. Probes never appeared at the undesignated end.

Procedure. The procedure was the same as in Experiment 1.

Design. The design was the same as in Experiment 1, with the following exceptions. The independent variables were (1) probe location (center or designated end), (2) rotation (present or absent), (3) translation (present or absent), and (4) growth (present or absent). Probe location and growth were run within subjects, whereas rotation and translation were run between subjects. The growth conditions were presented in separate blocks, in a counterbalanced order. In addition, the probe events were sorted into four categories of line length: less than $3.5^{\circ}$ of visual angle (360 pixels), between $3.5^{\circ}$ and $6.8^{\circ}$ (361 and 700 pixels), between $6.8^{\circ}$ and $9.3^{\circ}$ (701 and 960 pixels), and greater than $9.3^{\circ}$ (960 pixels). This comprised another within-subjects independent variable, line length (very short, short, long, or very long), in the analysis.

\section{Results}

An average of 11.5 probes were presented per experimental trial, resulting in an average of 577 probes per observer. Line-tracking accuracy averaged $96.7 \%$ across all observers. Only trials on which (1) both of the target lines were correctly identified and (2) no more than four false alarms were recorded were included in the probe detection analyses. Thus, an average of 3.81 trials, or 43.6 probe events, were excluded per observer. An average of 1.06 false alarms occurred per tracking trial, for trials included in the probe detection analyses.

Motion components analysis. The mean detection miss rates were submitted to a 2 (probe location) $\times 2$ (rotation) $\times 2$ (translation) $\times 2$ (growth) $\times 4$ (line length) mixed ANOVA. The main effect of probe location was significant $[F(1,44)=45.39, p<.001]$ : Miss rates were lower for probes occurring at centers $(M=.37, S D=.11)$ rather than ends $(M=.53, S D=.13)$. The main effect of line length was significant $[F(2.4,107.0)=21.06, p<$ $.001]$. There was also a significant interaction between probe location and line length $[F(2.6,114.6)=29.73$, $p<.001]$; this interaction can be seen in Figure 4. For end probes, miss rates increased as line length increased, but for center probes, miss rates remained constant as line length increased.

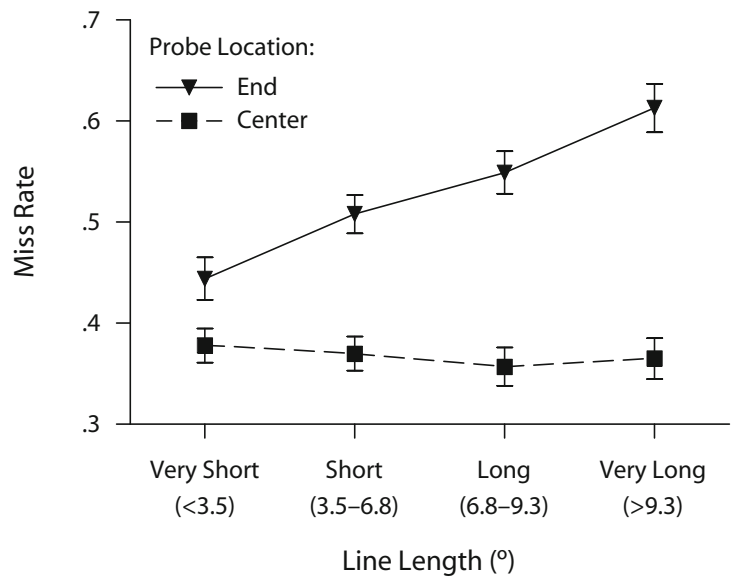

Figure 4. Miss rate as a function of line length and probe location in Experiment 3.

The main effect of translation was significant $[F(1,44)=4.29, p<.05]$, with miss rates higher when translation was present $(M=.48, S D=.09)$ than when it was absent $(M=.42, S D=.09)$. The main effect of rotation was not significant $[F(1,44)=0.00, p>.05]$, since miss rates did not differ when rotation was present $(M=$ $.45, S D=.09)$ from when it was absent $(M=.45, S D=$ .09 ). The main effect of growth was also not significant $[F(1,44)=0.07, p>.05]$; miss rates did not differ when growth was present $(M=.45, S D=.09)$ and absent $(M=$ $.45, S D=.10)$. There was a significant three-way interaction between probe location, line length, and translation $[F(2.6,114.6)=4.78, p<.01]$ : The magnitude of the difference between end and center miss rates increased more with line length when translation was absent than when it was present.

Importantly, there was no significant interaction between rotation and probe location $[F(1,44)=0.03, p>$ $.05]$. As can be seen in Figure 5A, the magnitude of the difference between end and center miss rates did not differ when rotation was present and absent. There was also no significant interaction between translation and probe location $[F(1,44)=0.35, p>.05]$. As can be seen in Figure $5 \mathrm{~B}$, the magnitude of the difference between end and center miss rates did not differ with translation present and translation absent. Finally, there was no significant interaction between growth and probe location $[F(1,44)=$ $0.53, p>.05]$. As can be seen in Figure 5C, the magnitude of the difference between end and center miss rates was equal for growth present and growth absent. There were no other significant interactions involving probe location.

Stationary conditions analysis. A separate ANOVA was conducted on the miss rates from only the stationary conditions, in order to investigate the occurrence of center bias with stationary lines. This was a 2 (probe location) $\times 4$ (line length) repeated measures ANOVA, which involved the 12 observers who viewed the stationary conditions. The main effect of probe location was significant $[F(1,11)=17.75, p<.01]$, with lower miss rates for 

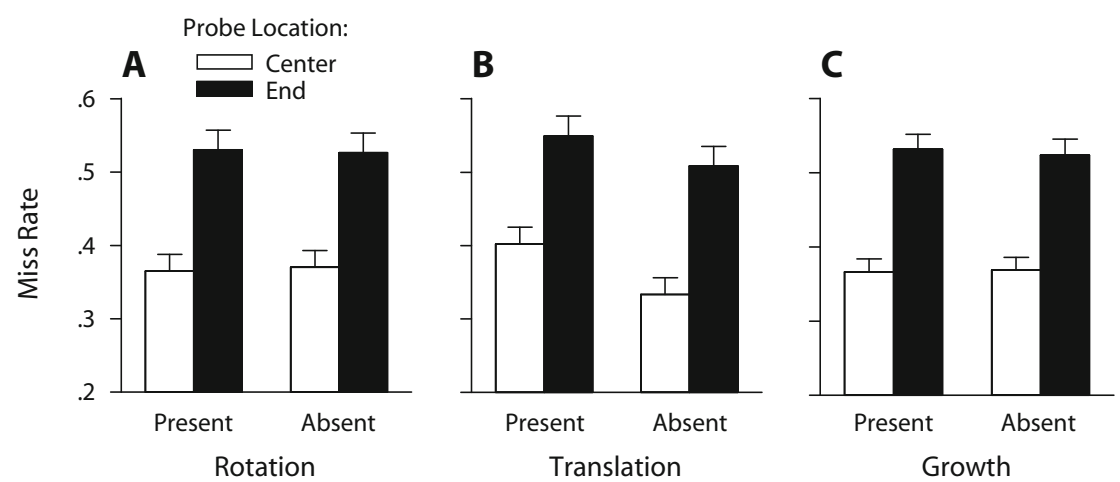

Figure 5. Miss rate as a function of three types of object motion-(A) rotation, (B) translation, (C) growth - and probe location in Experiment 3.

probes occurring at centers $(M=.27, S D=.11)$ than for those at ends $(M=.40, S D=.14)$. The main effect of line length was significant $[F(2.6,28.3)=13.53, p<.001]$, and there was also a significant interaction between probe location and line length $[F(2.1,23.6)=5.75, p<.01]$. For end probes, miss rates increased as line length increased, but for center probes, miss rates remained fairly constant as line length increased.

\section{Discussion}

In Experiment 3, the motion components analysis found that, overall, probe detection performance was better for centers than for ends of lines, even though there was no strategic advantage to attend to the center on the basis of probe probabilities. Crucially, this analysis also revealed an absence of interactions between rotation and probe location, translation and probe location, and growth and probe location. This indicates that translation, rotation, and size change motions do not affect the magnitude of the center bias; the attentional center bias occurs regardless of which types of motion are present.

The motion components analysis also revealed that as line length increased, detection performance declined at the endpoint, but remained constant at the center. This is the same pattern that was found in Experiment 1, when the center was the high-probability location. Taken together, these results suggest that when attention is concentrated at the center of an object (whether solely because of an inherent center bias, as in Experiment 3, or in part because of spatial probabilities, as in Experiment 1), the attention becomes increasingly concentrated at the center relative to the endpoints as object size increases.

The analysis of the stationary conditions found that probe detection performance was better at centers than at the ends of lines. This indicates that even when objects are stationary, attention is still concentrated at the centers of objects. This finding demonstrates that the center bias is not caused by the tracking of object motion. Alvarez and Scholl (2005) hypothesized that the center of an object is the focus of attention because the center is the most stable point. The finding that the center bias is no smaller for objects undergoing pure translation or objects remaining stationary, as compared with objects undergoing rotation or size changes, is evidence against this hypothesis. The findings in this experiment, however, are consistent with the hypothesis that the concentration of attention at the centers of stationary and moving objects is a manifestation of the visual system's general bias toward the centers of objects and scenes.

An additional finding of the analysis of stationary conditions was that line length interacted with probe location in a fashion similar to that in the overall motion components analysis. This demonstrates that the effect of line length on the attentional distribution is similar for stationary and for moving objects, at least when probes occur in multiple locations on the objects.

\section{GENERAL DISCUSSION}

The goals of this study were to examine the distribution of attention within objects in multiple-object scenes and to investigate the existence of an attentional bias toward the centers of objects.

The study had two primary results. The first of these, found in Experiment 1, is that the attentional distribution within individual moving objects is affected by two factors: (1) an inherent center bias and (2) prioritization by spatial probabilities. The finding of an attentional bias toward the centers of objects is consistent with the findings of Alvarez and Scholl (2005). Nonetheless, the attentional bias toward the centers of moving objects can be modulated by manipulating probe location probabilities. This is consistent with the literature showing that the visual system attentionally prioritizes spatial locations on the basis of the probabilities of a target appearing at these locations (e.g., Geng \& Behrmann, 2005; Prinzmetal et al., 2005). In fact, as found in Experiment 2, when probe locations are known with $100 \%$ certainty, the center bias is eliminated. This result indicates that it is not mandatory for attention to be concentrated at the center of a moving object in order to track the object.

Several previous studies have shown that target probabilities can prioritize attention to particular locations in space, and a few studies have shown that such spatial pri- 
oritizations can also occur within objects and object-like configurations. Shomstein and Yantis (2004) showed that spatial probabilities can prioritize attention to locations within objects in combination with object-based attention. Miller (1988) and Hoffmann and Kunde (1999) found that spatial probabilities can prioritize attention to locations defined by relative position within a configuration. The findings of the present study are novel, in that they show that attentional prioritizations on the basis of spatial probabilities can move along with a continuously moving object. Probabilities of probe appearance can prioritize attention not only to particular fixed locations in space; when the probes occur on an object, and the object is in motion, the attentional prioritizations accompany the object as it travels. This supports the idea that the frame of reference for attentional prioritization is, at least in some cases, object- rather than space-based.

However, because the spatial positions in this study were designated by color, another interpretation of the findings is that observers prioritize attention to the color of the high-probability locations, rather than to the highprobability spatial locations per se. An additional possibility is that observers prioritize attention not only to the high-probability location on each object, but to an extended "part" of the object, including the high-probability location and adjacent locations of the same color. Much previous research has shown that attention can be directed toward features, such as color (e.g., Shih \& Sperling, 1996; Vierck \& Miller, 2005; but see also Brawn \& Snowden, 1999), and toward individual parts of an object (e.g., Barenholtz \& Feldman, 2003; Reppa \& Leek, 2003; Vecera, Behrmann, \& Filapek, 2001). Some support for these possible explanations of the present results can be seen in Figure 2 from Experiment 1, which reveals a trend ( $p=$ .088 ) in the center high-probability condition toward better probe detection at the same-color end (which would be in the same "part" as the center) than at the different-color end. These possible interpretations need to be addressed in future research.

Another question of interest is whether the concentration of attention at the centers of objects occurs automatically. As maintained by many researchers, a process is strictly (or strongly) automatic only if two criteria are satisfied: (1) the load insensitivity criterion, meaning that the process is not hindered when task demands are increased, and (2) the intentionality criterion, meaning that the process is not subject to voluntary control (see, e.g., Turatto et al., 2000; Yantis \& Jonides, 1990). According to the intentionality criterion, the attentional center bias should not be affected by the probabilities of probes occurring in different locations on the object. However, Experiments 1 and 2 showed that the center bias is modulated by spatial probabilities and is eliminated when no uncertainty exists as to the probe location. This indicates that the allocation of greater attention to the centers of objects is not strictly automatic. Overall, these results suggest that perhaps the visual system uses a default attentional distribution in which the centers of objects are allocated more attention than other parts. However, this default distribution can be controlled by task demands, to create a context-dependent attentional distribution in which high-probability locations are allocated greater attention.

The second primary result of this study is that the attentional distribution is biased toward the center of objects even when objects are stationary, as well as when they are moving. Experiment 3 found that the addition of rotational, translational, and size-change motions did not affect the size of the attentional center bias. This result was unexpected, and is surprising, given that previous literature assumed that the attentional center bias was a result of tracking object motion (Alvarez \& Scholl, 2005). This finding is not consistent with Alvarez and Scholl's hypothesis that the center of an object is the focus of attention because it is the most stable point on the object. This result is more consistent with the idea that the center bias is a pervasive phenomenon in visual perception. This attentional bias toward the centers of moving and stationary objects could be related to the finding that saccades to stationary objects or arrays land near the centers of those objects or arrays (see, e.g., Vishwanath \& Kowler, 2003, 2004). It has been suggested that saccade landing locations are computed by averaging across all stimulated locations within the target object or array (e.g., Vishwanath \& Kowler, 2003, 2004). Zhou et al. (2006) suggested a similar explanation for their finding that attention is drawn toward the center of an array of objects. They proposed that averaging across all stimulated locations results in the center of the array being the most salient location in the "saliency map" of the scene that guides attention.

Perhaps the results of the present study can be understood by extending Zhou et al.'s (2006) idea. As well as the entire scene forming an array that is averaged and results in the greater salience of the center of the array, perhaps the parts of each individual object are also averaged. Averaging the locations of parts of an object will result in the greater salience of the center of the object. Thus, saliency will be heightened not only at the center of the scene, but also at the center of each object. In order for this explanation to account for attentional prioritization by spatial probabilities, the saliency map's averaging must not be a simple average of the spatial locations, but instead must be weighted by the probability of probes appearing at each location. Future research is needed to further elaborate and empirically test this model.

In summary, the present study supports the existence of an attentional bias toward the centers of objects in multiple-object scenes. Attention tends to concentrate at the centers of both stationary and moving objects, and the presence of motion does not affect the magnitude of this bias. However, the attentional distribution within objects in multiple-object scenes is also subject to prioritization by spatial probabilities. In fact, the center bias is eliminated when probes are certain to appear at only one particular location on each object. These results indicate that attentional prioritizations on the basis of spatial probabilities can move along with a continuously moving object. They also imply that the center concentration of attention is not a strictly automatic process. A possible explanation for the attentional center bias is proposed on the basis of feature averaging, both on the basis of objects and on the 
basis of the scene as a whole, with the averaging weighted by spatial probabilities.

\section{AUTHOR NOTE}

This research was supported by Grant 4-69018-05-496 from the Kentucky jurisdiction of the National Science Foundation's Experimental Program to Stimulate Competitive Research. Parts of this research were presented at the annual meetings of the Vision Sciences Society (Sarasota, Florida, May 2006) and the Psychonomic Society (Long Beach, California, November 2007). I thank Maureen Doyle for her great help in developing the computer program. For assistance with data collection and useful conversations, I thank Allison Adams, Amy Baker, Jamie Cole, Joshua Edwards, Philip Oliver, Christa Patterson, Amanda Pieratt, Nick Rudd, Jessica Stephens, Amy Vaughn, and Cassie Watkins. I also thank Brian Scholl for helpful conversation and Gilbert Remillard for valuable advice and discussions. Correspondence concerning this article should be addressed to C. S. Feria, Department of Psychology, One Washington Square, San Jose State University, San Jose, CA $95192-$ 0120 (e-mail: cary.feria@sjsu.edu).

\section{REFERENCES}

Alvarez, G. A., \& Scholl, B. J. (2005). How does attention select and track spatially extended objects? New effects of attentional concentration and amplification. Journal of Experimental Psychology: General, 134, 461-476.

Barenholtz, E., \& Feldman, J. (2003). Visual comparisons within and between object parts: Evidence for a single-part superiority effect. Vision Research, 43, 1655-1666.

BRawn, P., \& SNOWden, R. J. (1999). Can one pay attention to a particular color? Perception \& Psychophysics, 61, 860-873.

Chen, Z., \& CAVE, K. R. (2006). Reinstating object-based attention under positional certainty: The importance of subjective parsing. Perception \& Psychophysics, 68, 992-1003.

CoËFfÉ, C., \& O'REgan, J. K. (1987). Reducing the influence of nontarget stimuli on saccade accuracy: Predictability and latency effects. Vision Research, 27, 227-240

FindLAY, J. M. (1982). Global visual processing for saccadic eye movements. Vision Research, 22, 1033-1045.

Geng, J. J., \& Behrmann, M. (2005). Spatial probability as an attentional cue in visual search. Perception \& Psychophysics, 67, 1252-1268.

Grabowecky, M., Robertson, L. C., \& Treisman, A. (1993). Preattentive processes guide visual search: Evidence from patients with unilateral visual neglect. Journal of Cognitive Neuroscience, 5, 288-302.

He, P., \& Kowler, E. (1989). The role of location probability in the programming of saccades: Implications for "center-of-gravity" tendencies. Vision Research, 29, 1165-1181.

HoFFMANN, J., \& KUNDE, W. (1999). Location-specific target expectancies in visual search. Journal of Experimental Psychology: Human Perception \& Performance, 25, 1127-1141.

Horowitz, T. S., Birnkrant, R. S., Fencsik, D. E., Tran, L., \& Wolfe, J. M. (2006). How do we track invisible objects? Psychonomic Bulletin \& Review, 13, 516-523.

Keane, B. P., \& Pylyshyn, Z. W. (2006). Is motion extrapolation employed in multiple object tracking? Tracking as a low-level, nonpredictive function. Cognitive Psychology, 52, 346-368.

Liu, G., Austen, E. L., Booth, K. S., Fisher, B. D., Argue, R., RemPEL, M. I., \& ENNS, J. T. (2005). Multiple-object tracking is based on scene, not retinal, coordinates. Journal of Experimental Psychology: Human Perception \& Performance, 31, 235-247.

Miller, J. (1988). Components of the location probability effect in visual search tasks. Journal of Experimental Psychology: Human Perception \& Performance, 14, 453-471.

Morgan, M. J., Hole, G. J., \& Glennerster, A. (1990). Biases and sensitivities in geometrical illusions. Vision Research, 30, 1793-1810.
Prinzmetal, W., McCool, C., \& ParK, S. (2005). Attention: Reaction time and accuracy reveal different mechanisms. Journal of Experimental Psychology: General, 134, 73-92.

PyLyshyn, Z. W., \& Storm, R. W. (1988). Tracking multiple independent targets: Evidence for a parallel tracking mechanism. Spatial Vision, 3, 179-197.

REPPA, I., \& LEEK, E. C. (2003). The modulation of inhibition of return by object-internal structure: Implications for theories of object-based attentional selection. Psychonomic Bulletin \& Review, 10, 493-502.

Scholl, B. J., \& Pylyshyn, Z. W. (1999). Tracking multiple items through occlusion: Clues to visual objecthood. Cognitive Psychology, 38, 259-290.

Scholl, B. J., Pylyshyn, Z. W., \& Feldman, J. (2001). What is a visual object? Evidence from target merging in multiple object tracking. Cognition, 80, 159-177.

SHIH, S., \& SperLING, G. (1996). Is there feature-based attentional selection in visual search? Journal of Experimental Psychology: Human Perception \& Performance, 22, 758-779.

Shomstein, S., \& Yantis, S. (2002). Object-based attention: Sensory modulation or priority setting? Perception \& Psychophysics, 64, 41-51.

Shomstein, S., \& Yantis, S. (2004). Configural and contextual prioritization in object-based attention. Psychonomic Bulletin \& Review, 11, 247-253.

Suganuma, M., \& YokosaWA, K. (2006). Grouping and trajectory storage in multiple object tracking: Impairments due to common item motions. Perception, 35, 483-495.

Turatto, M., Benso, F., Facoetti, A., Galfano, G., Mascetti, G. G., \& UMiLTÀ, C. (2000). Automatic and voluntary focusing of attention. Perception \& Psychophysics, 62, 935-952.

vanMarle, K., \& Scholl, B. J. (2003). Attentive tracking of objects versus substances. Psychological Science, 14, 498-504.

Vecera, S. P., Behrmann, M., \& FilapeK, J. C. (2001). Attending to the parts of a single object: Part-based selection limitations. Perception \& Psychophysics, 63, 308-321.

VierCK, E., \& Miller, J. (2005). Direct selection by color for visual encoding. Perception \& Psychophysics, 67, 483-494.

Vishwanath, D., \& Kowler, E. (2003). Localization of shapes: Eye movements and perception compared. Vision Research, 43, $1637-1653$

Vishwanath, D., \& Kowler, E. (2004). Saccadic localization in the presence of cues to three-dimensional shape. Journal of Vision, $\mathbf{4}$, 445-458.

Viswanathan, L., \& Mingolla, E. (2002). Dynamics of attention in depth: Evidence from multi-element tracking. Perception, 31, $1415-1437$

Walthew, C., \& Gilchrist, I. D. (2006). Target location probability effects in visual search: An effect of sequential dependencies. Journal of Experimental Psychology: Human Perception \& Performance, 32, 1294-1301.

Whitaker, D., McGraw, P. V., Pacey, I., \& Barrett, B. T. (1996). Centroid analysis predicts visual localization of first- and secondorder stimuli. Vision Research, 36, 2957-2970.

WhitAKER, D., \& WALKER, H. (1988). Centroid evaluation in the vernier alignment of random dot clusters. Vision Research, 28, 777-784.

YANTIS, S. (1992). Multielement visual tracking: Attention and perceptual organization. Cognitive Psychology, 24, 295-340.

YANTIS, S., \& JonidES, J. (1990). Abrupt visual onsets and selective attention: Voluntary versus automatic allocation. Journal of Experimental Psychology: Human Perception \& Performance, 16, 121-134.

Zhou, X., Chu, H., Li, X., \& Zhan, Y. (2006). Center of mass attracts attention. NeuroReport, 17, 85-88.

(Manuscript received June 4, 2007; revision accepted for publication March 18, 2008.) 